\title{
A Bioprocessed Black Rice Bran Glutathione-Enriched Yeast Extract Protects Rats and Mice against Alcohol-Induced Hangovers
}

\author{
Sung Phil Kim¹, Ju Ryeong Lee1, Ki Sun Kwon1, Yeo Jin Jang1, Jeanman Kim¹, Keun Hyung Yu², \\ Sun Yeop Lee ${ }^{2}$, Mendel Friedman ${ }^{3 *}$ \\ ${ }^{1}$ STR Biotech Co., Ltd., Chuncheon, Republic of Korea \\ ${ }^{2}$ Department of Technical Development, Chuncheon Bioindustry Foundation, Chuncheon, Republic of Korea \\ ${ }^{3}$ Western Regional Research Center, Agricultural Research Service, U.S. Department of Agriculture, Albany, CA, USA \\ Email: *ksp1108@gmail.com, *mendel.friedman@usda.gov
}

How to cite this paper: Kim, S.P., Lee, J.R., Kwon, K.S., Jang, Y.J., Kim, J., Yu, K.H., Lee, S.Y. and Friedman, M. (2021) A Bioprocessed Black Rice Bran Glutathione-Enriched Yeast Extract Protects Rats and Mice against Alcohol-Induced Hangovers. Food and Nutrition Sciences, 12, 223-238.

https://doi.org/10.4236/fns.2021.123018

Received: February 8, 2021

Accepted: March 12, 2021

Published: March 15, 2021

Copyright $\odot 2021$ by author(s) and Scientific Research Publishing Inc. This work is licensed under the Creative Commons Attribution International License (CC BY 4.0).

http://creativecommons.org/licenses/by/4.0/ (c) (i) Open Access

\begin{abstract}
Human and animal alcohol-induced hangovers are caused by adverse effects of acetaldehyde formed in vivo by the enzymatic oxidation of ethyl alcohol to acetaldehyde. This study aims to determine the effect of the combination of a bioprocessed black rice bran (BRB-F) and glutathione-enriched yeast extract (GEYE) on hangovers as tested in rats and mice. Because analysis by HPLC showed that the content of the biologically active rice bran compound $\gamma$-oryzanol as well as of the antioxidant reduced glutathione were unaffected during the preparation of tablets containing $100 \mathrm{mg} / \mathrm{kg}$ of the bran formulation, the tablets were then administered orally to rats. The results showed decreased blood concentrations of both alcohol and acetaldehyde compared to the control group. Additional behavior experiments using the Rota-rod and wire tests in mice confirmed that the food formulation relieved hangover behavior caused by alcohol. It seems that the combination of BRB-F and GEYE can effectively control hangovers in rodents caused by alcohol intake. Mechanistic aspects of the hangover and anti-hangover effects of alcohol-derived acetaldehyde are similar to browning-type reactions between the aldehyde group of glucose and proteins, the antibiotic effects of cinnamaldehyde against pathogenic bacteria, the adverse effects of the heat-induced food toxin acrylamide, and the alkali-induced formation of the unnatural amino acid lysinoalanine.
\end{abstract}




\section{Keywords}

Alcohol, Acetaldehyde, Hangover Inhibition, Black Rice Bran, Rodents, Mechanisms

\section{Introduction}

Black rice bran contains biologically active compounds, including phenolics, flavonoids, and anthocyanins with potential health benefits [1]. In a series of published studies, we reported on the health-promoting properties of black rice brans bioprocessed (fermented) with other foods, including mushroom polysaccharides and mycelia, reviewed in [2]. To place the results of the present study in proper perspective, we will first briefly highlight the observed beneficial effects.

Using a panel of chemical, biochemical, and cell assays, Choi, et al. [3] demonstrated anti-allergic effects of five black rice bran water-ethanol $(70 \% \mathrm{v} / \mathrm{v})$ extracts. The anti-allergic effects were accompanied by modulation of the pro-inflammatory cytokines. Black rice bran from the LK1-3-6-12-1-1 cultivar was the most effective inhibitor in all assays. Feeding a standard diet with added $10 \%$ black rice bran significantly suppressed induced allergic contact dermatitis on the skin of mice [4]. By contrast, nonpigmented brown rice bran was ineffective. The skin healing effect was accompanied by a reduction of inflammatory biomarkers, suggesting the potential value of black rice bran as an anti-inflammatory and antiallergic food ingredient. Intraperitoneal or oral administration of a polysaccharide isolated from Lentinus edodes (Shiitake) mushroom mycelia bioprocessed with black rice bran also protected mice against necrosis of the liver caused by pathogenic Salmonella typhimurium bacteria through the activation of a macrophage-mediated immune response resulting from augmented immunity [5]. In a related study, Kim, et al. [6] found that the bioprocessed mushroom polysaccharide-black rice bran product also protected mice against endotoxemia (sepsis, septic shock), an inflammatory, virulent often lethal human disease, via stimulation of the immune system. Another investigation by Kim, et al. [7] reported that the antibiotic mechanism of a bioprocessed shiitake (Lentinus edodes) mushroom mycelia and black rice bran formulation against Salmonella enterica involves the phagocytosis of extracellular bacteria, autophagic capture of intracellular bacteria, and prevention of translocation of bacteria across the intestinal epithelial cells. The in vivo antimicrobial properties suggest that the formulation could serve as a functional antimicrobial food and medical antibiotic. A study by Choi, et al. [8] found that feeding tumor-bearing mice black and brown rice bran for 2 weeks reduced tumor mass by $35 \%$ and $19 \%$, respectively. The tumor regression was associated with increases in cytolytic activity of splenic natural killer cells and inhibition of angiogenesis, suggesting the possible value of the formulation as an anti-cancer functional food. 
Feeding tumor-bearing mice a standard diet for 2 weeks supplemented with the black rice bran component $\gamma$-oryzanol also significantly reduced tumor mass [9]. The mechanism of this tumor regression was also associated with the induction of natural killer cell activity, activation of macrophages, and inhibition of angiogenesis, suggesting the possible value of $\gamma$-oryzanol in cancer therapy. We also reported that turmeric and elm bark bioprocessed in mushroom mycelia media also exhibited in vivo anti-allergic and antibiotic health benefits [10] [11]. Because ethanol-derived acetaldehyde toxicity seems to be a major cause of human and animal hangovers, these multiple health-promoting benefits of black rice bran-based functional foods motivated us to consider determining if bioprocessed (fermented) black rice bran, containing the anti-cancer compound $\gamma$-oryzanol, combined with a yeast extract containing the antioxidant reduced glutathione would also inhibit ethanol-induced hangovers in rats and mice, the objective of the present study.

\section{Materials and Methods}

\subsection{Materials}

2-Propanol, acetic acid, trifluoroacetic acid (TFA), ethanol, and carboxymethyl cellulose (CMC) were purchased from Sigma-Aldrich (St. Louis, MO, USA). Potato dextrose agar medium (PDA) was purchased from Difco Laboratory (Detroit, MI, USA). Glutathione-enriched yeast extract was purchased from Zhejiang Senyo Biotech. Co., Ltd. (Huzhou City, China).

First, confirm that you have the correct template for your paper size. This template has been tailored for output on the custom paper size $(21 \mathrm{~cm} \times 28.5$ $\mathrm{cm})$.

\subsection{Cultivation of Mushroom Mycelia and Preparation of a Bioprocessed Polysaccharide (BRB-F)}

Lentinus edodes fungal mycelia were isolated from the mushroom fruitbody and cultured on PDA medium. The genetic identity of the fungus was confirmed by the Korean Center of Microorganisms (Seoul, Korea). The mycelium cultured in PDA media was inoculated into $50 \mathrm{~mL}$ of the liquid medium (Table 1). Incubation experiments were performed in $250 \mathrm{~mL}$ Erlenmeyer flasks for 5 days at $28^{\circ} \mathrm{C}$ on a rotary shaker $(120 \mathrm{rpm})$ and used to seed the main liquid culture. The main liquid medium contained black rice bran $(20 \mathrm{~g} / \mathrm{L})$ and dried soybean powder $(2$ $\mathrm{g} / \mathrm{L})$. Subsequently, the medium was treated with amylase and protease enzymes at $60^{\circ} \mathrm{C}$ for 60 minutes for enzymatic digestion of particulate matter containing carbohydrates and proteins. The culture was then adjusted to $\mathrm{pH} 6.0$ using $\mathrm{HCl}$, and then sterilized in the autoclave. Experiments on the main liquid culture were started by inoculating the inoculum (10\%) of pre-cultured mycelium using a $5 \mathrm{~L}$ fermenter (working volume of $3 \mathrm{~L}$ ) at $28^{\circ} \mathrm{C}$ and $150 \mathrm{rpm}$. After 7 days, the culture mass was crushed in a colloid mill (model PUC60 Hankook Power Technology System, Seoul, Korea). The powder was treated with $0.1 \mathrm{M}$ lactic acid for 
$60 \mathrm{~min}$, then treated with an enzyme mixture for cell wall lysis containing cellulose, hemi-cellulase, pectinase, glucanase, mannose, and arabinase at $50^{\circ} \mathrm{C}$ for 60 min. Subsequently, the acid-treated and enzyme-treated cultures were extracted with hot water at $90^{\circ} \mathrm{C}$ for $1 \mathrm{~h}$ and lyophilized with a solid material. Table 1 shows the composition of the liquid culture used to carry out the bioprocessing operation.

\subsection{Analysis of $\gamma$-Oryzanol and Reduced Glutathione in a Tablet and Pill Prototypes}

HPLC analysis was carried out according to the method described by the Shimazu Corporation [12], and Sutariya, et al. [13] with slight modification. For $\gamma$-oryzanol analysis, $0.5 \mathrm{~g}$ of sample was placed in $50 \mathrm{~mL}$ volumetric flask and dissolved with ethyl alcohol, ultrasonically extracted for 60 minutes, and allowed to cool to room temperature for 10 minutes. The extract was filtered using a 0.45 mm membrane filter. A Shimadzu HPLC system and SPD-M20A Diode Array Detector (Shimadzu, Kyoto, Japan) were used, and the analysis column was Shim-pack GIS CN $(150 \times 4.6 \mathrm{~mm}, 5 \mathrm{~mm}$, Shimadzu $)$. The mobile phase was analyzed in the isocratic mode for 15 minutes at a 5:95 ratio using isopropanol (A) and $1 \%$ acetic acid in hexane (B). The flow rate was $0.5 \mathrm{~mL}$ per minute, the temperature of the column oven was $30^{\circ} \mathrm{C}$, the detection wavelength was 320 $\mathrm{nm}$, and the injection volume of the sample was $20 \mathrm{~mL}$ (Table 2). For reduced glutathione analysis, $0.25 \mathrm{~g}$ of sample was placed in a $1000 \mathrm{~mL}$ volumetric flask and dissolved with $0.1 \%$ trifluoroacetic acid in water, stirred for 10 minutes, and filtered with a $0.45 \mathrm{~mm}$ membrane filter. The Shimadzu HPLC system and SPD-M20A Diode Array Detector (Shimadzu, Kyoto, Japan) were used, and the analysis column was Triart C18 $(250 \times 4.6 \mathrm{~mm}, 5 \mathrm{~mm}$, YMC). The mobile phase was analyzed in the isocratic mode for 30 minutes at a $95: 5$ ratio using $0.1 \%$ trifluoroacetic acid in water(A) and $0.1 \%$ trifluoroacetic acid in acetonitrile(B). The flow rate was $0.8 \mathrm{~mL}$ per minute, the temperature of the column oven was $30^{\circ} \mathrm{C}$, the detection wavelength was $214 \mathrm{~nm}$, and the injection volume of the sample was $20 \mathrm{~mL}$ (Table 3).

Table 1. Composition of the liquid medium used for L. edodes mycelia culture.

\begin{tabular}{cc}
\hline Ingredients & Amount (\%) \\
Glucose & 2 \\
Yeast extract & 0.5 \\
Soy peptone & 0.5 \\
$\mathrm{KH}_{2} \mathrm{PO}_{4}$ & 0.2 \\
$\mathrm{MgSO}_{4}$ & 0.05 \\
$\mathrm{FeSO}_{4}$ & 0.002 \\
\hline
\end{tabular}


Table 2. HPLC conditions for the analysis of $\gamma$-oryzanol.

\begin{tabular}{cccc}
\hline Instrument & \multicolumn{3}{c}{ Shimadzu HPLC } \\
\hline Column & \multicolumn{3}{c}{ Shimadzu Shim-pack CN Column $(150 \times 4.6 \mathrm{~mm}, 5 \mathrm{~mm})$} \\
Column Temp. & $30^{\circ} \mathrm{C}$ & \\
Mobile phase & Time (min) & A: 2 -propanol & B: $1 \%$ acetic acid, hexane \\
(Isocratic mode) & 0 & 5 & 95 \\
& 15 & 5 & 95 \\
Detector & & PDA $(320 \mathrm{~nm})$ & \\
Flow rate & $0.5 \mathrm{~mL} / \mathrm{min}$ \\
Injection volume & $20 \mathrm{~mL}$ \\
\hline
\end{tabular}

Table 3. HPLC conditions for the analysis of reduced glutathione.

\begin{tabular}{|c|c|c|c|}
\hline Instrument & \multicolumn{3}{|c|}{ Shimadzu HPLC } \\
\hline Column & \multicolumn{3}{|c|}{ YMC Triart C18 column $(250 \times 4.6 \mathrm{~mm}, 5 \mathrm{~mm})$} \\
\hline Column Temp. & & $30^{\circ} \mathrm{C}$ & \\
\hline Mobile phase & Time (min) & A: $0.1 \%$ TFA, water & B: $0.1 \%$ TFA, ACN \\
\hline \multirow[t]{2}{*}{ (Isocratic mode) } & 0 & 5 & 95 \\
\hline & 15 & 5 & 95 \\
\hline Detector & & PDA $(214 \mathrm{~nm})$ & \\
\hline Flow rate & & $0.8 \mathrm{~mL} / \mathrm{min}$ & \\
\hline Injection volume & & $20 \mathrm{~mL}$ & \\
\hline
\end{tabular}

\subsection{Preparation of Anti-Hangover Pills and Tablets}

Anti-hangover pills and tablets were produced by the Natural Way Co., Ltd. (Pocheon, Korea) and CNS Pharm Korea Co., Ltd. (Eumseong, Korea) companies, respectively. The pills were prepared by mixing BRB-F (20\% of total weight) and GEYE (13.3\% of total weight) with an excipient. The mixture was used to prepare the pills with the aid of a pill-making machine and then dried in a dryer. The tablets were prepared by mixing BRB-F (28.8\% of total weight) and GEYE (19.2\% of total weight) with excipients, the mixture was granulated, and then tableted using a tablet press.

\subsection{Alcohol and Acetaldehyde Scavenging Assay In Vivo}

Animal experiments using rats were carried out according to the method described by Lee, et al. [14] and Yang, et al. [15]. Pathogen-free male SD rats, 7 weeks old, were purchased from DBL Co. Ltd. (Eumseong, Korea). The rats were housed in a stainless-steel cage under a $12 \mathrm{~h} \mathrm{light/dark} \mathrm{cycle} \mathrm{with} \mathrm{a} \mathrm{temperature}$ range of $23^{\circ} \mathrm{C} \pm 3^{\circ} \mathrm{C}$ and relative humidity of $50 \% \pm 10 \%$. Rats were fed the pelletized normal commercial chow diet (Cat. No. 5L79, Orient Bio., Seongnam, Korea) and tap water ad libitum for 1 week after arrival for acclimation. Acclimatized rats were arbitrarily divided into the following five groups (for deter- 
mination of dosage, $\mathrm{n}=10$ ) or three groups (for experiment of prototype, $\mathrm{n}=$ 10 ), avoiding any intergroup difference in body weight. Rats were orally administered samples for each concentration in 0.5\% CMC after fasting for 18 hours, and $40 \%$ ethanol was orally administered at a concentration of $3 \mathrm{~g} / \mathrm{kg} 30$ minutes after sample administration. After 0 hour, 30 minutes, 1 hour, 3 hours, and 5 hours, blood was collected from the tail vein, and after 8 hours rats were sacrificed, and blood was collected by heart puncture. The collected blood was left on ice for 30 minutes and centrifuged at $2000 \times g$ for 30 minutes to separate the serum. The amount of alcohol and acetaldehyde in the serum was measured according to the manufacturer's instruction using an alcohol assay kit (Cell Biolabs, Inc. San Diego, CA) and acetaldehyde assay kit (BioAssay Systems, Hayward, CA). All experimental procedures were carried out in accordance with the guidelines of the Institutional Animal Care and Use Committee at Chuncheon Bioindustry Foundation (CBF IACUC No. 2020-002).

\subsection{Mouse Behavior Test}

Animal experiments using mice were carried out according to the method described by Karadayian, et al. [16]. Pathogen-free male ICR mice, 6 weeks old, were purchased from DBL Co. Ltd. (Eumseong, Korea). The mice were housed in a stainless-steel cage under a $12 \mathrm{~h}$ light/dark cycle with a temperature range of $23^{\circ} \mathrm{C} \pm 3^{\circ} \mathrm{C}$ and relative humidity of $50 \% \pm 10 \%$. Mice were fed the pelletized normal commercial chow diet (Cat. No. 5L79, Orient Bio.) and tap water ad libitum for 1 week after arrival for acclimation. Motor control and balance were tested using accelerating Rota-rod (B.S Technolab Inc. Seoul. Korea). Animals were acclimated to the Rota-rod twice a day for 2 days before the experiment and the running time and the falling frequency was measured before $40 \%$ alcohol administration at a concentration of $3 \mathrm{~g} / \mathrm{kg}$ and after $0.5 \mathrm{~h}, 1 \mathrm{~h}, 3 \mathrm{~h}, 5 \mathrm{~h}$, and $8 \mathrm{~h}$, respectively. Running time was evaluated by measuring the time the mouse exercised without falling at $4 \mathrm{rpm}$, and the falling frequency was evaluated by measuring the number of times the mouse fell while exercising at $4 \mathrm{rpm}$ for 30 minutes. Limb strength and balance were evaluated through a wire test. Mice were adapted to a wire ( $55 \mathrm{~cm}$ wide, $2 \mathrm{~mm}$ thick metallic wire) 2 days before the experiment. The mice were then hung on the wire after alcohol administration. The latency time and falling frequency was then measured at $0.5 \mathrm{~h}, 1 \mathrm{~h}, 3 \mathrm{~h}, 5 \mathrm{~h}$, $8 \mathrm{~h}$, respectively. Latency time was evaluated by measuring the time taken until the mouse first fell off the wire, and the falling frequency was evaluated by measuring the number of times the mouse fell from the wire for 5 minutes.

\subsection{Statistical Analysis}

Results are expressed as the mean \pm SD of three independent experiments. Significant differences between means were determined by the one-way ANOVA test followed by Duncan's multiple range test using the Statistical Analysis Software package SAS (Cary, NC, USA). $p<0.05$ is regarded as significant. 


\section{Results}

\subsection{Alcohol and Acetaldehyde Scavenging-Like Activity by Different Ratios of BRB-F and GEYE}

To confirm the scavenging-like activity of BRB-F and GEYE against alcohol and acetaldehyde, scavenging-like activity was measured using alcohol and acetaldehyde assay kits and methods described in the literature [17] [18] [19]. The results in Table 4 show that the scavenging-like activity against alcohol and acetaldehyde by BRB-F alone was very low. Although the GEYE treatment did increase the scavenging-like activity, the increase for the combination of BRB-F and GEYE with a ratio of 3:2 was significantly greater: 4.74-fold for acetaldehyde scavenging and 4.75 -fold for alcohol scavenging.

\subsection{Blood Alcohol and Acetaldehyde Scavenging Activity Induced by the BRB-F and GEYE Mixture}

The effect of the mixture of BRB-F and GEYE on alcohol and acetaldehyde metabolism in the body was confirmed using a rat hangover animal model. Alcohol was administered 30 minutes after the administration of the BRB-F and GEYE complex. The measured blood alcohol and acetaldehyde concentrations confirmed that concentration-dependent decreases in alcohol and acetaldehyde concentrations were observed at all administered doses shown in Figure 1(A) and Figure 2(A) and Figure 1(B) and Figure 2(B). The data show that, based on calculated areas under the curve (AUC), administration of the BRB-F and GEYE mixtures at 50,100, and $200 \mathrm{mg} / \mathrm{kg}$ resulted in $10.7 \%, 19.3 \%$, and $30.9 \%$ dose-dependent inhibitory effects, respectively. The corresponding calculation for acetaldehyde (Figure 1(B), and Figure 2(B)) also shows dose-dependent inhibitory effects of $7.9 \%, 16.9 \%$, and $23.2 \%$, respectively. These observations indicate the combination is effective in reducing the blood levels of the two compounds that are associated with producing hangovers in rats.

Table 4. In vitro alcohol and acetaldehyde scavenging activity for different ratios of BRB-F and GEYE.

\begin{tabular}{ccc}
\hline Sample $(10 \mathrm{mg} / \mathrm{mL})$ & $\begin{array}{c}\text { Alcohol scavenging-like activity } \\
(\%)\end{array}$ & $\begin{array}{c}\text { Acetaldehyde scavenging-like activity } \\
(\%)\end{array}$ \\
\hline BRB-F only & $13.9 \pm 1.2^{\mathrm{e}}$ & $7.23 \pm 0.52^{\mathrm{e}}$ \\
GEYE only & $42.5 \pm 3.8^{\mathrm{d}}$ & $14.9 \pm 1.2^{\mathrm{d}}$ \\
BRB-F 1: GEYE 1 & $57.2 \pm 2.0^{\mathrm{c}}$ & $37.1 \pm 3.2^{\mathrm{a}}$ \\
BRB-F 2: GEYE 1 & $62.2 \pm 5.7^{\mathrm{bc}}$ & $25.9 \pm 1.6^{\mathrm{b}}$ \\
BRB-F 1: GEYE 2 & $73.5 \pm 5.0^{\mathrm{a}}$ & $16.8 \pm 2.8^{\mathrm{cd}}$ \\
BRB-F 3: GEYE 2 & $68.3 \pm 4.3^{\mathrm{a}}$ & $34.3 \pm 2.5^{\mathrm{a}}$ \\
BRB-F 2: GEYE 3 & $70.1 \pm 3.5^{\mathrm{b}}$ & $19.6 \pm 1.5^{\mathrm{c}}$
\end{tabular}

Data are expressed as the mean \pm SD of triplicate experiments. Values sharing a common letter are not significantly different at $\mathrm{p}<0.05$. 
(B)
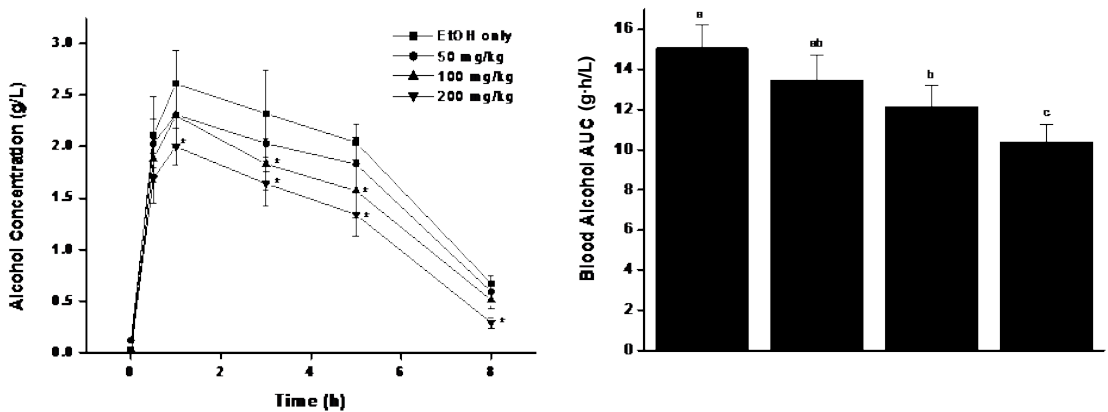

Figure 1. Rat blood alcohol scavenging activity of 3:2 mixture of BRB-F and GEYE. Changes in blood alcohol concentration (A) and blood alcohol concentration AUC (B) over time after alcohol administration. ${ }^{*} \mathrm{p}<0.05$ for means $(\mathrm{n}=10)$ of each treatment versus the ethyl alcohol only control for each time; bars sharing a common letter are not significantly different at $\mathrm{p}<0.05$.
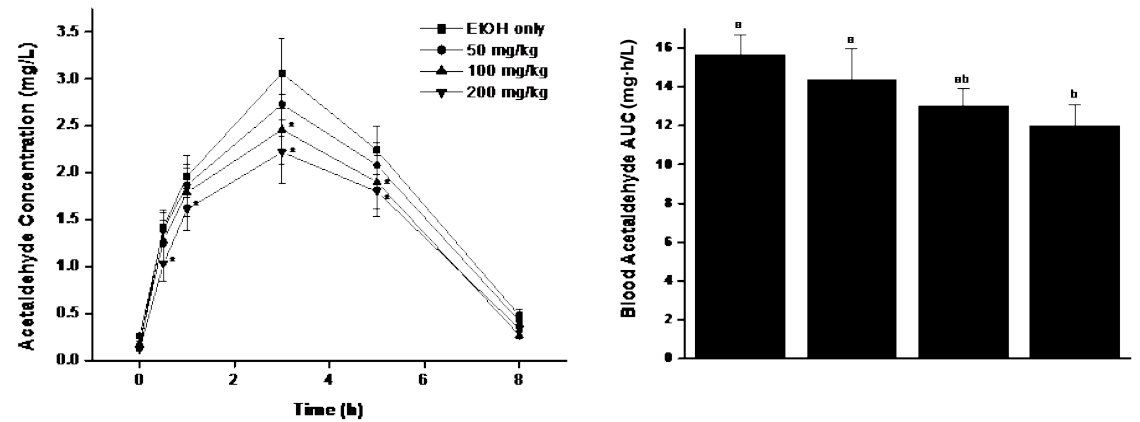

Figure 2. Blood acetaldehyde scavenging activity according to the dosage of BRB-F and GEYE mixture. Changes in blood acetaldehyde concentration (A) and blood acetaldehyde concentration AUC (B) over time after alcohol administration. ${ }^{*} \mathrm{p}<0.05$ for means of each treatment with ethyl alcohol as the control for each time; bars sharing a common letter are not significantly different at $\mathrm{p}<0.05$.

\section{3. $\gamma$-Oryzanol and Reduced Glutathione Analysis of Tablet and Pill Formulations}

Samples were analyzed for $\gamma$-oryzanol and reduced glutathione using the HPLC conditions shown in Table 2 and Table 3. Representative chromatograms are shown in Figure 3. The samples included BRB-F, GEYE, BRB-F and GEYE in a ratio of 3:2 in a mixed powder made before the formulation of pills and tablets, which were also analyzed. The results show that the mixture contained 101\% and $100 \%$ of added $\gamma$-oryzanol and reduced glutathione, respectively. Surprisingly, the content of $\gamma$-oryzanol and reduced glutathione in the pills was only $62 \%$ and $43.3 \%$, respectively, of the quantities added before the pills were made, thus showing significant losses during pill production. This was not the case for the tablets, which have $101 \%$ content of both $\gamma$-oryzanol and reduced glutathione that corresponds to the original amounts added to the BRB-F powder. These results show that the concentrations of the two compounds decreased during the manufacture of the pills but not the tablets. We therefore used the 
tablets for the in vivo studies.

\subsection{Blood Alcohol and Acetaldehyde Scavenging Activity of the Tablets}

We investigated the effect of administering the tablets on the blood alcohol and acetaldehyde concentrations at a dosage of $208 \mathrm{mg} / \mathrm{kg}$ (containing $100 \mathrm{mg} / \mathrm{kg}$ of BRB-F and GEYE mixture). The results show that both blood alcohol and acetaldehyde concentrations decreased significantly compared to the control group (Figure 4(A), Figure 5(A)). The calculated values using AUC confirmed that alcohol and acetaldehyde levels decreased by $38.3 \%$ and $36.9 \%$, respectively (Figure 4(B), Figure 5(B)). These findings indicate that the use of tablets containing the developed anti-hangover products might be a convenient way to test their efficacy in human trials.
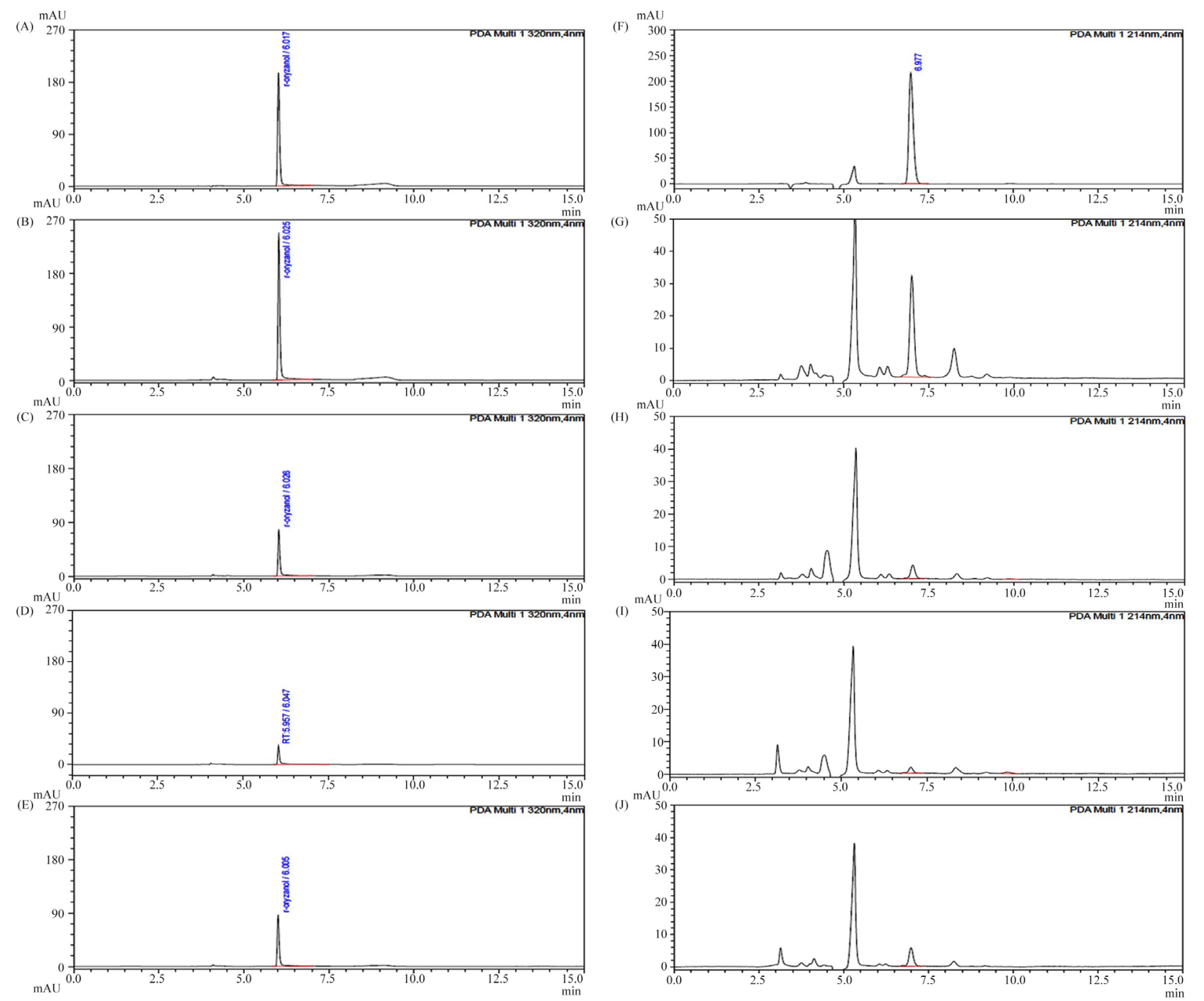

Figure 3. HPLC chromatograms for $\gamma$-oryzanol analysis: $15.63 \mathrm{ppm} \gamma$-oryzanol standard (A), BRB-F only (B), BRB-F and GEYE mixture powder before formulation (C), pill type formulation (D) and tablet type formulation (E). Chromatograms for reduced glutathione analysis: $250 \mathrm{ppm}$ reduced glutathione standard (F), GEYE only (G) BRB-F and GEYE mixture powder before formulation (H), pill type formulation (I), and tablet type formulation (J). 

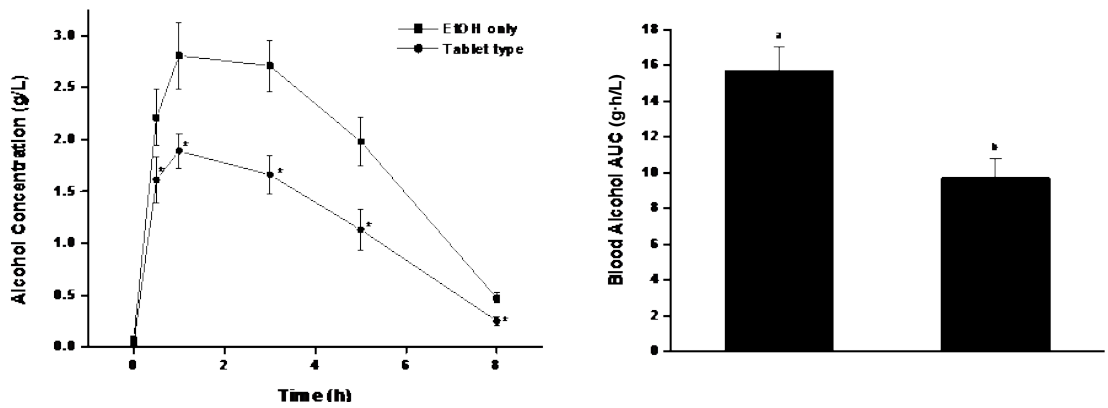

Figure 4. Blood alcohol scavenging activity of anti-hangover tablets. Changes in blood alcohol concentration (A) and blood alcohol concentration AUC (B) over time after alcohol administration. ${ }^{*} \mathrm{p}<0.05$ for means of each treatment versus ethyl alcohol as the control for each time period. Bar graphs sharing a common letter are not significantly different at $\mathrm{p}<0.05$.
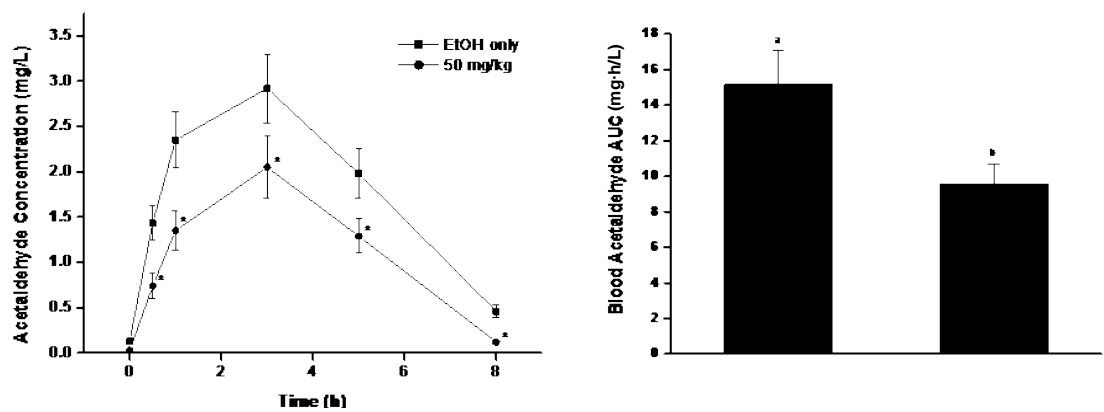

Figure 5. Blood acetaldehyde scavenging activity of anti-hangover tablets. Changes in blood acetaldehyde concentration (A) and blood acetaldehyde concentration AUC (B) over time after alcohol administration. ${ }^{\star} \mathrm{p}<0.05$ for means of each treatment versus ethyl alcohol as the control for each time periods. Bars sharing a common letter are not significantly different at $\mathrm{p}<0.05$.

\subsection{Anti-Hangover Tablets Relieve Alcohol-Induced Behavioral Disorder in Mice}

To confirm the described in vivo anti-hangover effects in rats, we evaluated the effect of the orally administered tablets in mice. The tablets were administered 30 minutes before alcohol administration at a concentration of $208 \mathrm{mg} / \mathrm{kg}$ (containing $100 \mathrm{mg} / \mathrm{kg}$ of the BRB-F and GEYE mixture). The effect on the behavior of the treated mice and controls without the tablets was evaluated using the known Rota-rod test and wire behavior tests. The results using the Rota-rod test in Figure 6 show an increase in exercise duration and a decrease in the falling frequency compared the ethanol control group without the tablets. With the wire test, the results show that the time it took for the first drop increased and the falling frequency decreased (Figure 7) compared to the ethanol control group. Taken together, the two results suggest that the tablets have an inhibitory effect on the alcohol-induced psychomotor alteration in the mice. The psychomotor data seem to reinforce the anti-hangover effect of the therapeutic tablets. This aspect also merits confirmation in clinical studies with human patients. 
(A)

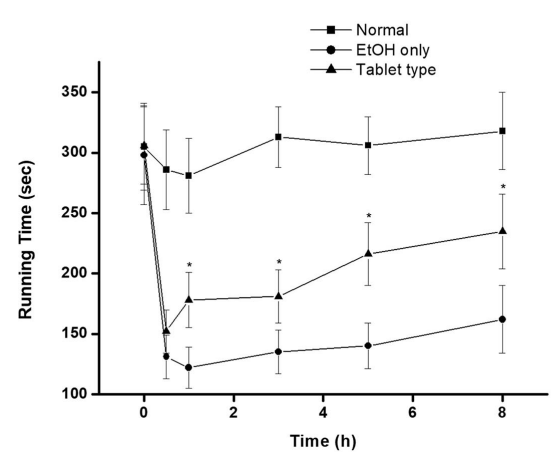

(B)

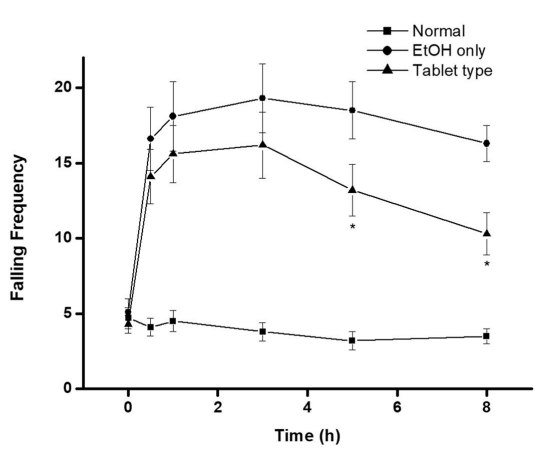

Figure 6. The time-dependent effect of improving hangover behavior by the anti-hangover tablets using in the Rota-rod behavior test (A). This figure shows the time the mice spent exercising without dropping at $4 \mathrm{rpm}$. Figure (B) shows the falling frequency of the mice during exercise for 30 minutes at $4 \mathrm{rpm} .{ }^{*} \mathrm{p}<0.05$ for means $(\mathrm{n}=10$ of each treatment compared to ethyl alcohol control group of mice for each time period.

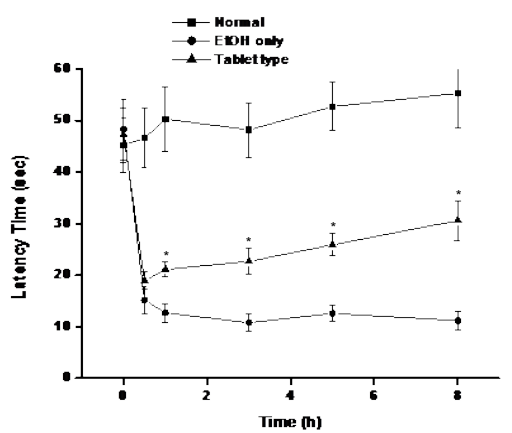

(B)

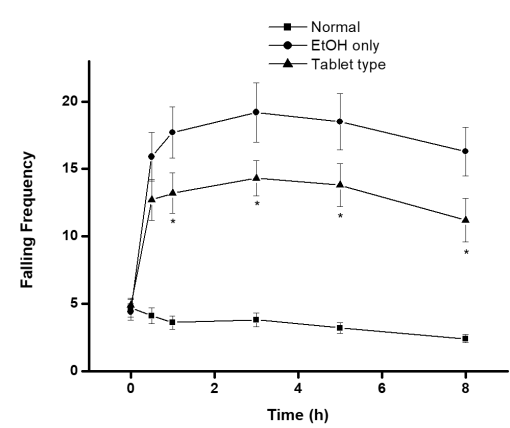

Figure 7. (A) The effect of the anti-hangover tablets on improving hangover behavior using the wire test and the time it took for the first falling. (B) The falling frequency of the mice while hanging for 5 minutes. ${ }^{*} \mathrm{p}<0.05$ for means of each treatment compared to the ethanol control group.

\section{Discussion}

\subsection{Related Studies}

Here, we will briefly mention several reported studies of the anti-hangover effects of some food-based and other natural products. An investigation by Lee, Song, Kim, Joo, Park, Jeon, Shin, Park, Lee, Ly, Kim, Lee and Kim [14] of the effects of a preparation of combined glutathione-enriched yeast (GEY) and rice embryo/soybean (RES) extracts (20:1), GEY/RES, on experimentally induced ethanol hangover in male Sprague-Dawley rats found that GEY/RES reduced the blood concentrations of alcohol and acetaldehyde by modulating alcohol-metabolizing enzymes and by exhibiting antioxidant activity, suggesting that it might be a promising candidate for improving an alcoholic hangover. Bajpai, et al. [20] reported that heat-treated cucumber juice detoxified alcohol in experimental rats. A human study by Mammen, et al. [21] found that clove bud polyphenols alleviated changes in inflammation and oxidative stress biomarkers 
associated with binge drinking. Two reviews describe the use of natural products to prevent hangovers [22] [23]. The authors of the second review concluded that the available evidence suggests that several products are capable of significantly improving some, but not all, of the symptoms related to alcohol hangover and that further research is necessary to develop clinically effective hangover treatments.

\subsection{Mechanistic Aspects}

Acetaldehyde is a toxic byproduct of alcohol metabolism found in the blood following alcohol consumption. Adverse effects associated with nonenzymatic covalent protein binding by acetaldehyde are most likely associated with the modification of structural and functional proteins and DNA [24] [25]. Although we do not know the exact mechanism that governs the anti-hangover effect of the new food product, the beneficial effects most likely occur by alleviating systemic inflammatory reactions caused by alcohol consumption, stimulation of the immune system [26] [27], and by facilitating glutathione activity by reducing oxidized glutathione (GSSG) to the antioxidative reduced form (GSH). GSH is a cysteine-containing tripeptide that can react rapidly with acetaldehyde in plasma and tissues alleviating adverse hangover effects as described below for L-cysteine.

Modeling and analytical studies by Lewis, et al. [28] suggest that a protonated Schiff base structure generated from acetaldehyde in vivo may be central to pathophysiological outcomes because it determines the structure of the stable covalent adduct formed. Also noteworthy are reports that the $\mathrm{SH}$-containing amino acid L-cysteine alleviates the alcohol-related hangover symptoms of nausea, headache, stress and anxiety in humans [29]. We reported on the beneficial effects of L-cysteine in preventing food browning in fresh and dehydrated potatoes and in apples [30] [31], the reduction of adverse effects of acrylamide in frog embryos [32] [33], the inhibition of formation of the unnatural amino acid lysinoalanine in proteins [34] as well as on the antibiotic effects of cinnamaldehyde [35]. The effects of L-cysteine are most likely the result of more rapid competitive nucleophilic addition reactions of its $\mathrm{SH}$ groups with the aldehyde groups of acetaldehyde and the conjugated double bond of acrylamide and dehydroalanine that react to form analogous alkylation products. Because the pKa value of NAC and consequently its nucleophilic reactivity of the ionized $\mathrm{SH}$ group of $\mathrm{N}$-acetyl-L-cysteine $\left(\mathrm{HSCH}_{2} \mathrm{CH}\left(\mathrm{NHCOCH}_{3}\right) \mathrm{COOH}, \mathrm{NAC}\right)$ is greater than that of L-cysteine [36], it would be of interest to determine if NAC [37] and GSH in which cysteine is part of a tripeptide [38] might exhibit greater anti-hangover activity than L-cysteine in rodents and humans.

\section{Conclusion}

This investigation has shown that a food-based product prepared by mixing bioprocessed black rice bran containing the bioactive compound $\gamma$-oryzanol and a glutathione-containing yeast extract inhibited the ethyl alcohol oxidation to ace- 
taldehyde in vitro and in rodents and the behavioral disorders in mice caused by alcohol consumption. The combined effects in rats and mice demonstrate suppression of alcohol-induced hangovers by the new food product. The results suggest the potential value of the new functional food product to ameliorate hangover-related adverse effects in humans. This finding complements our above-mentioned studies of several other health benefits of bioprocessed black rice bran-containing formulations. Therefore, it is likely that the described new functional food, a black rice bran containing product, might, in addition to anti-hangover properties, also have potential for use against a broad range of other diseases. Moreover, it also seems that the mechanism of the anti-hangover effects could be analogous to previously described mechanisms of food browning and its prevention, in vivo inhibition of adverse effects of the food processing induced toxin acrylamide and the amino acid lysinoalanine, as well antibiotic effects of cinnamaldehyde. Finally, it would also be useful if clinical trials could determine if the black rice bran product might help protect individuals against excessive (abusive) alcohol consumption that causes liver cirrhosis and cancer [39].

\section{Acknowledgements}

We thank In Jue Yun for technical support of the analysis of $\gamma$-oryzanol and glutathione and Carol Levin for facilitating the preparation of the manuscript.

This research was financially supported by the Ministry of Small and Medium-sized Enterprises (SMEs) and Startups (MSS), Korea, under the "Regional Enterprise Open-Innovative Voucher Program (R\&D, P0010861)" supervised by the Korea Institute for Advancement of Technology (KIAT).

\section{Conflicts of Interest}

The authors declare no conflicts of interest regarding the publication of this paper.

\section{References}

[1] Ghasemzadeh, A., Karbalaii, M.T., Jaafar, H.Z.E. and Rahmat, A. (2018) Phytochemical Constituents, Antioxidant Activity, and Antiproliferative Properties of Black, Red, and Brown Rice Bran. Chemistry Central Journal, 12, Article No.17. https://doi.org/10.1186/s13065-018-0382-9

[2] Friedman, M. (2016) Mushroom Polysaccharides: Chemistry and Antiobesity, Antidiabetes, Anticancer, and Antibiotic Properties in Cells, Rodents and Humans. Foods, 5, 80. https://doi.org/10.3390/foods5040080

[3] Choi, S.P., Kang, M.Y., Koh, H.J., Nam, S.H. and Friedman, M. (2007) Antiallergic Activities of Pigmented Rice Bran Extracts in Cell Assays. Journal of Food Science, 72, S719-S726. https://doi.org/10.1111/j.1750-3841.2007.00562.x

[4] Choi, S.P., Kim, S.P., Kang, M.Y., Nam, S.H. and Friedman, M. (2010) Protective Effects of Black Rice Bran against Chemically-Induced Inflammation of Mouse Skin. Journal of Agricultural and Food Chemistry, 58, 10007-10015. https://doi.org/10.1021/jf102224b 
[5] Kim, S.P., Park, S.O., Lee, S.J., Nam, S.H. and Friedman, M. (2014) A Polysaccharide Isolated from the Liquid Culture of Lentinus edodes (Shiitake) Mushroom Mycelia Containing Black Rice Bran Protects Mice against Salmonellosis through Up-Regulation of the Th1 Immune Reaction. Journal of Agricultural and Food Chemistry, 62, 2384-2391. https://doi.org/10.1021/jf405223q

[6] Kim, S.P., Park, S.O., Lee, S.J., Nam, S.H. and Friedman, M. (2013) A Polysaccharide Isolated from the Liquid Culture of Lentinus edodes (Shiitake) Mushroom Mycelia Containing Black Rice Bran Protects Mice against a Salmonella Lipopolysaccharide-Induced Endotoxemia. Journal of Agricultural and Food Chemistry, 61, 10987-10994. https://doi.org/10.1021/jf403173k

[7] Kim, S.P., Lee, S.J., Nam, S.H. and Friedman, M. (2018) The Composition of a Bioprocessed Shiitake (Lentinus edodes) Mushroom Mycelia and Rice Bran Formulation and Its Antimicrobial Effects against Salmonella enterica subsp. enterica Serovar Typhimurium Strain SL1344 in Macrophage Cells and in Mice. BMC Complementary and Alternative Medicine, 18, Article No. 322.

https://doi.org/10.1186/s12906-018-2365-8

[8] Choi, S.P., Kim, S.P., Nam, S.H. and Friedman, M. (2013) Antitumor Effects of Dietary Black and Brown Rice Brans in Tumor-Bearing Mice: Relationship to Composition. Molecular Nutrition \& Food Research, 57, 390-400. https://doi.org/10.1002/mnfr.201200515

[9] Kim, S.P., Kang, M.Y., Nam, S.H. and Friedman, M. (2012) Dietary Rice Bran Component $\Gamma$-Oryzanol Inhibits Tumor Growth in Tumor-Bearing Mice. Molecular Nutrition \& Food Research, 56, 935-944. https://doi.org/10.1002/mnfr.201200057

[10] Kim, S.P., Lee, S.J., Nam, S.H. and Friedman, M. (2016) Elm Tree (Ulmus parvifolia) Bark Bioprocessed with Mycelia of Shiitake (Lentinus edodes) Mushrooms in Liquid Culture: Composition and Mechanism of Protection against Allergic Asthma in Mice. Journal of Agricultural and Food Chemistry, 64, 773-784.

https://doi.org/10.1021/acs.jafc.5b04972

[11] Kim, S.P., Lee, S.J., Nam, S.H. and Friedman, M. (2017) Turmeric Bioprocessed with Mycelia from the Shiitake Culinary-Medicinal Mushroom Lentinus edodes (Agaricomycetes) Protects Mice against Salmonellosis. International Journal of $\mathrm{Me}$ dicinal Mushrooms, 19, 363-376. https://doi.org/10.1615/IntJMedMushrooms.v19.i4.70

[12] Shimazu Corporation (2017) Shim-Pack HPLC Column Guidebook. 41, C190-E206A.

[13] Sutariya, V., Wehrung, D. and Geldenhuys, W.J. (2012) Development and Validation of a Novel RP-HPLC Method for the Analysis of Reduced Glutathione. Journal of Chromatographic Science, 50, 271-276. https://doi.org/10.1093/chromsci/bmr055

[14] Lee, H.S., Song, J., Kim, T.M., Joo, S.S., Park, D., Jeon, J.H., et al. (2009) Effects of a Preparation of Combined Glutathione-Enriched Yeast and Rice Embryo/Soybean Extracts on Ethanol Hangover. Journal of Medicinal Food, 12, 1359-1367. https://doi.org/10.1089/jmf.2008.1367

[15] Yang, H.J., Kim, M.J., Kang, E.S., Kim, D.S. and Park, S. (2018) Red Mulberry Fruit Aqueous Extract and Silk Proteins Accelerate Acute Ethanol Metabolism and Promote the Anti-Oxidant Enzyme Systems in Rats. Molecular Medicine Reports, 18, 1197-1205. https://doi.org/10.3892/mmr.2018.9017

[16] Karadayian, A.G., Mac Laughlin, M.A. and Cutrera, R.A. (2012) Estrogen Blocks the Protective Action of Melatonin in a Behavioral Model of Ethanol-Induced Hangover in Mice. Physiology \& Behavior, 107, 181-186.

https://doi.org/10.1016/j.physbeh.2012.07.003 
[17] Oh, T., Kwon, M., Yu, J.S., Jang, M., Kim, G.H., Kim, K.H., et al. (2020) Ent-Peniciherqueinone Suppresses Acetaldehyde-Induced Cytotoxicity and Oxidative Stress by Inducing ALDH and Suppressing MAPK Signaling. Pharmaceutics, 12, 1229. https://doi.org/10.3390/pharmaceutics12121229

[18] Hayashida, Y., Hironaka, N. and Katayama, S. (2016) Effects of Solmach on Alcohol Hangover Model in Mice. Nihon Arukoru Yakubutsu Igakkai Zasshi, 51, 109-117.

[19] Zhang, Y.J., Wang, F., Zhou, Y., Li, Y., Zhou, T., Zheng, J., et al. (2016) Effects of 20 Selected Fruits on Ethanol Metabolism: Potential Health Benefits and Harmful Impacts. International Journal of Environmental Research and Public Health, 13, 399. https://doi.org/10.3390/ijerph13040399

[20] Bajpai, V.K., Kim, N.H., Kim, J.E., Kim, K. and Kang, S.C. (2016) Protective Effect of Heat-Treated Cucumber (Cucumis sativus L.) Juice on Alcohol Detoxification in Experimental Rats. Pakistan Journal of Pharmaceutical Sciences, 29, 1005-1009.

[21] Mammen, R.R., Natinga Mulakal, J., Mohanan, R., Maliakel, B. and Illathu Madhavamenon, K. (2018) Clove Bud Polyphenols Alleviate Alterations in Inflammation and Oxidative Stress Markers Associated with Binge Drinking: A Randomized Double-Blinded Placebo-Controlled Crossover Study. Journal of Medicinal Food, 21, 1188-1196. https://doi.org/10.1089/jmf.2017.4177

[22] Wang, F., Li, Y., Zhang, Y.J., Zhou, Y., Li, S. and Li, H.B. (2016) Natural Products for the Prevention and Treatment of Hangover and Alcohol Use Disorder. Molecules, 21, 64. https://doi.org/10.3390/molecules21010064

[23] Jayawardena, R., Thejani, T., Ranasinghe, P., Fernando, D. and Verster, J.C. (2017) Interventions for Treatment and/or Prevention of Alcohol Hangover: Systematic Review. Human Psychopharmacology, 32, e2600. https://doi.org/10.1002/hup.2600

[24] Oppolzer, D., Barroso, M. and Gallardo, E. (2016) Bioanalytical Procedures and Developments in the Determination of Alcohol Biomarkers in Biological Specimens. Bioanalysis, 8, 229-251. https://doi.org/10.4155/bio.15.240

[25] Rogers, C.M., Simmons Iii, R.H., Fluhler Thornburg, G.E., Buehler, N.J. and Bochman, M.L. (2020) Fanconi Anemia-Independent DNA Inter-Strand Crosslink Repair in Eukaryotes. Progress in Biophysics and Molecular Biology, 158, 33-46.

[26] van de Loo, A., van Schrojenstein Lantman, M., Mackus, M., Scholey, A. and Verster, J.C. (2018) Impact of Mental Resilience and Perceived Immune Functioning on the Severity of Alcohol Hangover. BMC Research Notes, 11, 526. https://doi.org/10.1186/s13104-018-3659-0

[27] van de Loo, A., Mackus, M., van Schrojenstein Lantman, M., Kraneveld, A.D., Brookhuis, K.A., Garssen, J., et al. (2018) Susceptibility to Alcohol Hangovers: The Association with Self-Reported Immune Status. International Journal of Environmental Research and Public Health, 15, 1286.

https://doi.org/10.3390/ijerph15061286

[28] Lewis, J., Smith, B.A., Oakes, H., Holman, R.W. and Rodnick, K.J. (2019) New Evidence for the Diversity of Mechanisms and Protonated Schiff Bases Formed in the Non-Enzymatic Covalent Protein Modification (NECPM) of HbA by the Hydrate and Aldehydic Forms of Acetaldehyde and Glyceraldehyde. Cogent Biology, 5, Article No. 1584955. https://doi.org/10.1080/23312025.2019.1584955

[29] Eriksson, C.J.P., Metsälä, M., Möykkynen, T., Mäkisalo, H., Kärkkäinen, O., Palmén, M., et al. (2020) L-Cysteine Containing Vitamin Supplement Which Prevents or Alleviates Alcohol-Related Hangover Symptoms: Nausea, Headache, Stress, and Anxiety. Alcohol and Alcoholism, 55, 660-666.

https://doi.org/10.1093/alcalc/agaa082 
[30] Friedman, M., Molnár-Perl, I. and Knighton, D.R. (1992) Browning Prevention in Fresh and Dehydrated Potatoes by SH-Containing Amino Acids. Food Additives \& Contaminants, 9, 499-503. https://doi.org/10.1080/02652039209374103

[31] Molnár-Perl, I. and Friedman, M. (1990) Inhibition of Browning by Sulfur Amino Acids. Part 3. Apples and Potatoes. Journal of Agricultural and Food Chemistry, 38, 1652-1656. https://doi.org/10.1021/jf00098a006

[32] Williams, J.R., Rayburn, J.R., Cline, G.R., Sauterer, R. and Friedman, M. (2014) The Potential Protective Effect of L-Cysteine against the Toxicity of Acrylamide and Furan in Exposed Xenopus laevis Embryos: An Interaction Study. Journal of Agricultural and Food Chemistry, 62, 7927-7938. https://doi.org/10.1021/jf5013743

[33] Friedman, M. (2015) Acrylamide: Inhibition of Formation in Processed Food and Mitigation of Toxicity in Cells, Animals, and Humans. Food \& Function, 6, 1752-1772. https://doi.org/10.1039/C5FO00320B

[34] Friedman, M. (1999) Chemistry, Biochemistry, Nutrition, and Microbiology of Lysinoalanine, Lanthionine, and Histidinoalanine in Food and Other Proteins. Journal of Agricultural and Food Chemistry, 47, 1295-1319. https://doi.org/10.1021/jf981000+

[35] Friedman, M. (2017) Chemistry, Antimicrobial Mechanisms, and Antibiotic Activities of Cinnamaldehyde against Pathogenic Bacteria in Animal Feeds and Human Foods. Journal of Agricultural and Food Chemistry, 65, 10406-10423. https://doi.org/10.1021/acs.jafc.7b04344

[36] Friedman, M. (1973) The Chemistry and Biochemistry of the Sulfhydryl Group in Amino Acids, Peptides, and Proteins. Pergamon Press, Oxford, England.

[37] Mocelin, R., Marcon, M., D’Ambros, S., Herrmann, A.P., da Rosa Araujo, A.S. and Piato, A. (2018) Behavioral and Biochemical Effects of N-Acetylcysteine in Zebrafish Acutely Exposed to Ethanol. Neurochemical Research, 43, 458-464. https://doi.org/10.1007/s11064-017-2442-2

[38] Karadayian, A.G., Malanga, G., Czerniczyniec, A., Lombardi, P., Bustamante, J. and Lores-Arnaiz, S. (2017) Free Radical Production and Antioxidant Status in Brain Cortex Non-Synaptic Mitochondria and Synaptosomes at Alcohol Hangover Onset. Free Radical Biology \& Medicine, 108, 692-703. https://doi.org/10.1016/j.freeradbiomed.2017.04.344

[39] Beers, M.H. (2006) The Merck Manual of Diagnosis and Therapy. 18th Edition, Merck Research Laboratories, Whitehouse Station, NJ. 